\title{
La Visión de la Nanotecnología para las Radiocomunicaciones en los próximos años. Una perspectiva desde la Academia.
}

\section{The Vision of Nanotechnology for the Radiocommunications in the coming years. A perspective from the Academy.}

\author{
Andrés Felipe García ${ }^{1}$, L. Betancur ${ }^{2}$
}

\begin{abstract}
Resumen
En los últimos años, la nanotecnología ha marcado un hito en la evolución de las comunicaciones, lo que ha permitido el desarrollo de nuevas aplicaciones y la estandarización de nuevos materiales en una escala nanométrica llamada nanomateriales. Algunos de ellos son el grafeno y sus derivados como los nanotubos de carbono y algunos compuestos como los metamateriales cuyas propiedades y características de tipo electrónico y físico son totalmente compatibles, permitiendo una fusión fácil con las telecomunicaciones. Y es precisamente lo que se pretende mediante este artículo; proporcionar una perspectiva desde la academia para identificar algunos tipos de nanomateriales que nos planteamos algunas preguntas como ¿qué tipo de materiales son?, ¿qué propiedades tienen?, ¿qué clasificación poseen?, ¿cuáles son algunas de las aplicaciones más importantes en el campo de las telecomunicaciones?, y ¿qué desarrollos y aplicaciones hay actualmente? Así, entramos explorando las nanocomunicaciones.
\end{abstract}

Palabras clave: Nanotecnología; nanocomunicaciones; nanoreceptor; grafeno; nanoantennas.

\begin{abstract}
In recent years, nanotechnology has marked a milestone in the communications evolution, which has allowed the development of new applications and standardization of new materials to a nanometer scale called nanomaterials. Some of them are the graphene and its derivatives as carbon nanotubes and some compounds as the metamaterials whose properties and characteristics of electronic and physical type are fully compatible, allowing easy merging with telecommunications. And it is precisely what is intended by this article: provide a perspective from the academy to identify some kinds of nanomaterials and we resolve some questions like ¿what kind of materials are? ¿what properties do it have? ¿what classification has it? ¿what are some of the most important applications in the field of telecommunications? and $¿$ what developments are there currently. Thus, we enter exploring the nanocommunications.
\end{abstract}

Keywords: Nanotechnology; nanocommunications; nanoreceptor; graphene; nanoantennas.

\section{INTRODUCCIÓN}

Desde hace ya una década aproximadamente, la nanotecnología se ha ido convirtiendo en uno de los complementos principales de otras ciencias, en torno a la investigación, el desarrollo y las aplicaciones industriales. Permitiendo así la reestructuración molecular de algunos elementos hasta llevarlos a una escala nanométrica. La nanotecnología no se clasifica o se limita a una sola tecnología o enfoque científico, todo lo contrario, se extiende hacía un conjunto multidisciplinario de diseño de procesos, de medición, de modelados, de caracterización y producción de materiales, dispositivos, aplicaciones, sistemas de control y diversos conceptos de tipo físico, químico, biológico y electrónico, donde su característica principal es el tamaño (Abal,2005).

El Instituto Foresight en Estados Unidos (Foresight Institute, 2014), ha clasificado la nanotecnología como un conjunto de tecnologías emergentes, donde la estructura de la materia es controlada a una escala nanométrica cuyo propósito está en producir nuevos materiales y dispositivos que incluyan propiedades útiles y únicas (Ramsden,2009).

${ }^{1}$ Universidad Pontificia Bolivariana. andresf.garciare@upb.edu.co, $\mathrm{MsC}, \mathrm{PhD}(\mathrm{c})$.

${ }^{2}$ Universidad Pontificia Bolivariana. leonardo.betancur@upb.edu.co, MsC, PhD. 
A su vez la NNI (National Nanotechnology Initiative,2001) ha definido la nanotecnología como la aplicación responsable de investigar y desarrollar tecnología que involucre estructuras que estén sobre un rango aproximado entre 1 y $100 \mathrm{~nm}$ (nanómetros), frecuentemente hacía una precisión atómica o molecular para la creación e implementación de estructuras, aparatos y sistemas que contengan propiedades y funciones novedosas debido a su escala de dimensiones nanométricas. Y con la habilidad para controlar o manipular sobre la escala atómica.

Posteriomente se destacan algunas investigaciones en el sector de las nanocomunicaciones; propiamente en el diseño, la fabricación y operación de las nanoantenas y los nanosensores. Generando un aporte significativo al identificar los beneficios y propiedades por la implementación de diversos metamateriales (Gou, et al,. 2011).

Actualmente en la fusión de las telecomunicaciones con la nanotecnología, los desarrollos en las nanocomunicaciones han incluido nanocompuestos, producto de procesos paralelos con otros nanomateriales que a su vez contienen ciertas propiedades piezoeléctricas y cuya estructura molecular es posible modificarla, facilitando entonces el modelado y el prototipaje de diseños que antes no era posible materializarlos.

Este trabajo se enfoca en la revisión y exploración del estado del arte en el campo de las nanocomunicaciones, teniendo en cuenta algunas aplicaciones, características, propiedades y tipos de redes que giran entorno a estructuras nanómetricas, materiales especiales y demás componentes. Es por ello que el capítulo II, se clasifica en dos componentes (A y B). En el componente A se se encuentran algunas generalidades en términos de nanotecnología. En el componente B se relacionan temas como el grafeno y los metamateriales, sus propiedades estructurales, térmicas y eléctricas. Posteriormente en el capítulo III, el enfoque se dirige a las aplicaciones en las nanocomunicaciones, incluyendo temas como las nanoantenas y los nanotubos de grafeno. Sus aplicaciones, tipos de redes y de sensores de nanotubos de carbono. Finalmente, el capítulo IV se enfoca en algunos avances a nivel de las nanocomunicaciones y su clasificación se divide en dos componentes (A y B). El componente A se dirige a los osciladores mecánicos de grafeno y el componente $\mathrm{B}$ a las comunicaciones cuánticas en la nanoescala.

\section{MARCO TEÓRICO Y ESTADO DEL ARTE}

\section{A. Generalidades}

La evolución que ha tenido la nanotecnología en los últimos cuatro años ha permitido nuevos desarrollos, soluciones y aplicaciones en el frente energético, biomédico, biológico, textil, robótico, industrial y de las telecomunicaciones, donde la fusión entre la nanotecnología y las comunicaciones la han transformado en las nanocomunicaciones.

A su vez los materiales aplicados en otros frentes de la nanotecnología operan igual en las nanocomunicaciones, elementos como el grafeno, el cuarzo y los nanotubos de carbono son algunos de los más utilizados en la construcción de nanocomponentes o nanodispotivos de tipo electrónico y de las comunicaciones.

Mediante el diseño, construcción y aplicación de nuevos nanodispositivos de comunicación se logran objetivos importantes como:

- Optimizar el rendimiento de operación de los componentes en una escala real.

- Garantizar un enlace de comunicación constante y preciso. - Transmisión y recepción en zonas de difícil acceso.

Debido a las propiedades que ofrecen los diferentes nanomateriales es posible llevar a cabo un proceso de síntesis, más llamado, aproximación "Bottom-up" (de abajo a arriba), donde los bloques constituyentes se configuran y se agrupan mediante estrategias controladas (Bartolomé, et al,. 2007), permitiendo así, obtener resultados con base en modelos previos. Se podrán visualizar a continuación algunos tipos y propiedades de los nano y meta materiales más comunes para desarrollos y aplicaciones en el ámbito de las nanocomunicaciones.

\section{B. Nanomateriales y sus propiedades de interés en las nanocomunicaciones}

\section{- El Grafeno}

Este material es un alotrópo, es decir; posee estructuras químicas diferentes del carbono. Un teselado hexagonal plano de seis átomos de carbono y de enlaces covalentes que se forman a partir de la superposición de los híbridos $s p^{2}$ de los carbonos enlazados y de un solo átomo (García, 2010) y (Moon, et al,. 2011). Esta particularidad en 
conjunto con técnicas especializadas posibilita consolidar capas de grafeno supremamente delgadas. Algunas de las propiedades más relevantes del grafeno son:

- Posee conductividad térmica, eléctrica y elástica (deformable en su estructura).

- En comparación con la fibra de carbono contiene mucha más flexibilidad manteniéndose igual de ligero.

- No presenta resultados negativos ante la radiación ionizante.

- Efecto Joule bajo o mínimo (es el calentamiento al conducir electrones).

- Reporta consumos mínimos de electricidad en comparación al silicio.

- Se convierte en un generador de electricidad al estar en contacto con la luz solar.

- Posee transparencia, resistencia e impermeabilidad.

- Abundante y económico (Moon, et al,. 2011), lo que minimiza los obstáculos para el desarrollo y la fabricación de componentes o aplicaciones a base de éste.

En la Figura 1 se visualiza un símil de la flexibilidad y la transparencia que ofrece un encapsulamiento de grafeno, mediante la alteración de su estructura molecular. Este tipo de estructuras conlleva al diseño de los SWCNT y MWCNT.

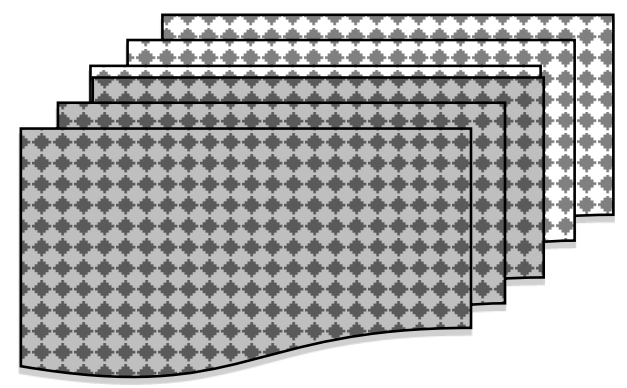

Figura. 1. Estructura tipo sandwich entre varios paneles de grafeno debido a la alteración de su estructura molecular.

\section{- Propiedades estructurales de los nanotubos de carbono}

Los nanotubos de carbono por sus siglas en inglés (CNT, carbon nanotubes) son alotrópos del carbono y un nanotubo de carbono de pared simple (SWCNT, single wall carbon nanotube), véase Figura 2 (b)), llega a aproximarse a una lámina de grafeno enrollada, es decir; una capa monoatómica de grafito cuya estructura se asemeja a un panel, véase Figura 2 (a)) con al menos un extremo como una media esfera compuesta por estructuras hexagonales y pentagonales de carbono. En la Figura 2 se presentan las diferentes capas que se conforman con las síntesis del grafeno: (a) hoja simple, (b) pared simple, (c) pared múltiple.

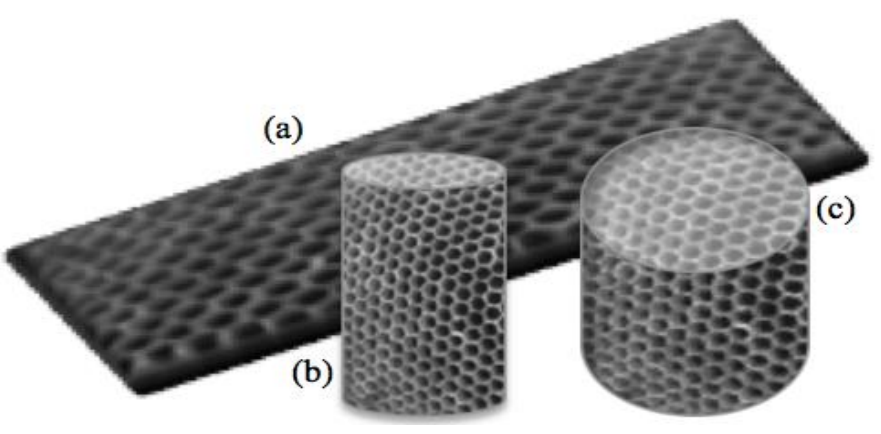

Figura. 2. (a) Hoja de grafeno, (b) SWCNT, (c) MWCNT.

En cuanto a los nanotubos de carbono de pared múltiple (MWCNT, multiple wall carbon nanotubes), véase Figura 2(c)), como su nombre lo indica, su estructura consiste en múltiples capas concéntricas de grafito enrolladas sobre sí mismas con el fin de formar una especie de tubo. La separación entre celdas de un MWCNT, está dada por la distancia de van der Waals entre las capas de grafeno en grafito $y$ se puede aproximar a $\delta=0,34 \mathrm{~nm}$ (Boudenot, 2008).

\section{- Propiedades térmicas de los nanotubos de carbono}

Lo que marca realmente las propiedades térmicas de los nanotubos de carbono es su conductividad térmica excepcional, ya que los MWCNT se encuentran entre los 600 y los $3000 \mathrm{Wm}^{-1} \mathrm{~K}^{-1}$. Los SWCNT entre los $2500 \mathrm{y}$ los 6600 $\mathrm{Wm}^{-1}$.

Y una película de CNT en abundancia posee entre 15 y $250 \mathrm{Wm}^{-1}$ en temperaturas superiores a los $100{ }^{\circ} \mathrm{k}$; posicionándolos en una escala de materiales nunca antes medidos (Boudenot, 2008). En la Tabla 1 se establece que los MWCNT y los SWCNT poseen mayor conductividad 
térmica respecto a otros materiales en su estado natural debido a estructura molecular reformada. Así mismo en la Figura 3 se visualiza dicha conductividad térmica, la cual aumenta con la temperatura, y es catalogada como un comportamiento clásico de los materiales semiconductores (Atsuko, et al,.2017) y (Bernholc, et al,.2002).

Los diferentes resultados producto de los experimentos que se han realizado basados en (Atsuko, et al,. 2017) y (Boudenot, 2008), destacan que la resistencia eléctrica disminuye linealmente unas cuantas centésimas cada vez que aumenta la temperatura en el nanotubo con un coeficiente térmico de resistencia variable disminuyendo hasta un $1 \%$ por grado. De esta manera un gradiente de temperatura a lo largo del nanotubo de carbono puede llegar a generar una diferencia de potencial debido a la dispersión que posee la carga como portadora, de una temperatura caliente a frío. Dando lugar a cierta difusión de energía termoeléctrica con un aumento de temperatura igual a los $80 \mu V K^{-1}$ a temperatura ambiente para los mismos $14 \mathrm{~nm}$ de diámetro de un MWCNT. En la Figura 3 se visualiza el incremento de conductividad térmica de los SWCNT respecto a la temperatura. Es decir, en la síntesis es posible llegar a capas sumamente delgadas, favoreciendo el aumento en la conductividad térmica.

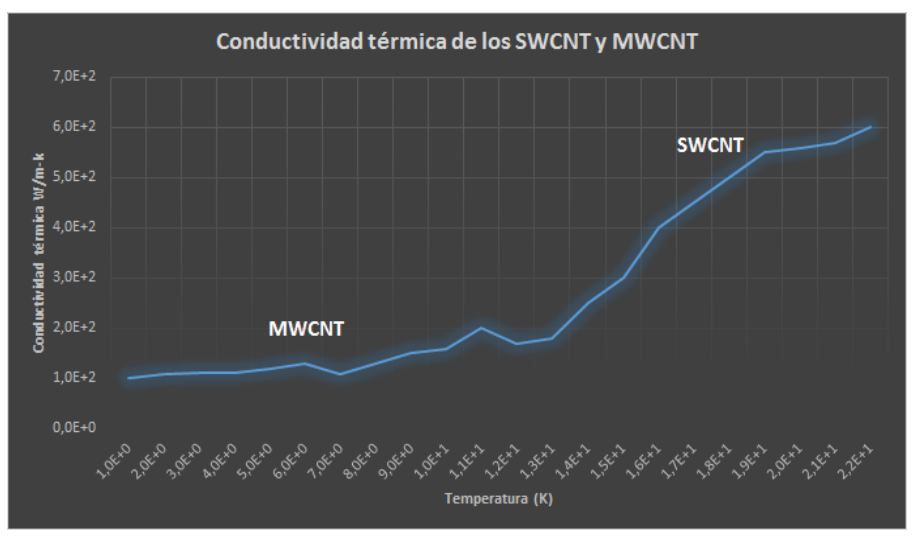

Figura. 3. Conductividad térmica de los SWNT y MWNT en función de la temperatura.

\section{- Propiedades eléctricas de los nanotubos de carbono}

Las propiedades eléctricas de los materiales conductores se modifican cuando se reduce su tamaño hacía una escala nanométrica. En la fabricación de un cable su dimensión de la sección transversal es comparable con la longitud de onda del electrón en la mecánica cuántica de Fermi, donde el alambre forma esencialmente una guía de onda de modo único para las ondas de electrones. Por lo tanto, en un conductor de una sola dimensión como un nanotubo, los electrones son libres para moverse a lo largo de la longitud del nanotubo y no en una dirección transversal. Por esto la distribución de la corriente es efectivamente unidimensional (Boudenot, 2008) y Scaccabarozzi, et al., 2017).

\section{- Los metamateriales}

Dentro de la categoría de materiales especiales, se encuentran los metamateriales que a su vez poseen diferentes beneficios, propiedades y aplicaciones tanto a nivel electrónico como de las telecomunicaciones. Los metamateriales están formados por estructuras periódicas o no periódicas de "átomos artificiales" o "partículas", que tienen un tamaño de escala de sublongitud de onda (Cui, 2012). Además, el diseño flexible de las partículas artificiales individuales, los arreglos factibles de tales partículas y la alta anisotropía hacen posible controlar las propiedades del material como se desee (Speech, 2012).

A través de los metamateriales se logra establecer una permitividad efectiva y permeabilidad, que no es posible encontrar en la naturaleza. Por lo tanto, poseen características únicas, tanto con fenómenos físicos inusuales (como la únicas, tanto con fenómenos físicos inusuales (como la refracción negativa, la capa de invisibilidad, ilusión óptica, etc.) o un rendimiento superior de los materiales naturales (Cui, 2012). 
Tabla 1. Comparación de conductividades térmicas entre nanotubos de carbón y materiales.

\begin{tabular}{|cccccccccc|}
\hline \multicolumn{7}{|c|}{ COMPARACIÓN DE CONDUCTIVIDAD TÉRMICA ENTRE NANOTUBOS DE CARBONO Y MATERIALES UNITARIOS } \\
\hline Materiales unitarios & Germanio & Silicio & Aluminio & Oro & Cobre & Diamante & MWCNT & SWCNT \\
\hline Conductividad térmica $\left(\mathrm{Wm}^{-1} \mathrm{~K}^{-1}\right)$ & 60 & 148 & 237 & 317 & 485 & 1000 & $2000-3000$ & $2500-6600$ \\
\hline
\end{tabular}

La categorización de los metamateriales se puede extender en metamateriales para aplicaciones de microondas y sus homólogos; en cristales, nanocristales y cuasicristales. Y estos en: metamateriales homogéneos, aleatorios y no homogéneos. Una de las clasificaciones más relevantes de los metamateriales se conforma en:

- Por la anisotropía artificial que contienen.

- Quirales y con encubrimiento.

- Sintonizables y no lineales.

- Por el índice de refracción negativa.

- Dieléctricos artificiales.

- Magnéticos artificales.

- Por el índice de refracción negativa.

En la Figura 4 se visualiza el modelo de un láser semiconductor a una frecuencia de THz, con emisión de haces con divergencia mucho más pequeñas respecto a las fuentes de láser en $\mathrm{THz}$ convencionales. Su $\lambda_{0}$ equivale a la longitud de fabricación, por lo que los patrones de los metamateriales son esculpidos directamente en la faceta del dispositivo.

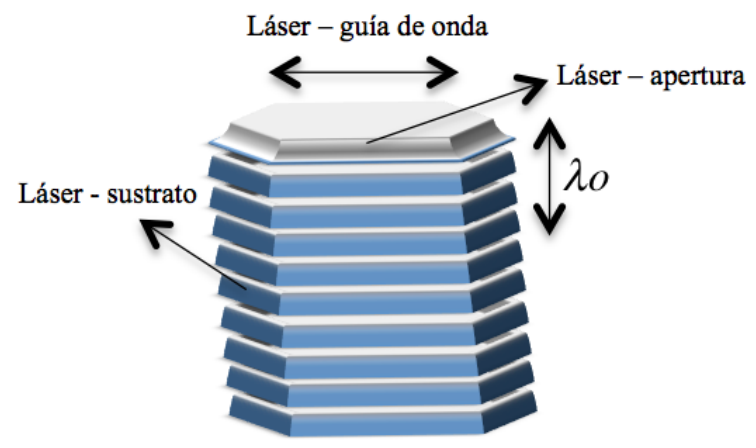

Figura. 4. Ejemplo de un semiconductor laser en THz construído a partir de capas de metamateriales. 


\section{APLICACIONES EN LAS NANOCOMUNICACIONES}

Las antenas tradicionales y versátiles ideales para diversas aplicaciones son las de alambre. Éste es un simple dispositivo donde se relacionan la mayoría de los mecanismos a nivel de radiación y de estructuración como en un dipolo. Su típica configuración se compone de dos cables conductores, con una longitud de $\frac{\lambda}{4}$ como se visualiza en la Figura 5 (Boudenot, 2008).

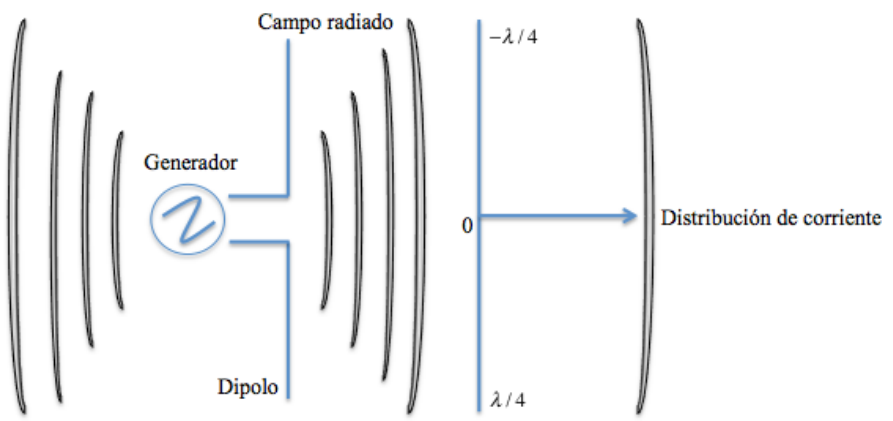

Figura. 5. Distribución de corriente y campo radiado en el espacio libre de un dipolo de media longitud de onda.

La distribución de corriente en el cable conductor puede considerarse unidimensional, cuya variación en el tiempo generará un campo electromagnético radiado en el espacio circundante. De acuerdo a las ecuaciones de Maxwell, estas conducen a la relación clásica entre la variación de la corriente $\mathrm{I}(\mathrm{z})$ y el campo radiado E $\theta$ en el espacio de campo lejano donde $\eta$ equivale a la impedancia característica del espacio libre, $\mathrm{k}$ a la constante de propagación, $\mathrm{L}$ a la longitud del dipolo, $\mathrm{r}$ a los radios y $\theta$ a las coordenadas de los ángulos de elevación (Boudenot, 2008) y (Burke, et al,. 2004). Un ejemplo de ello se visualiza en la siguiente ecuación:

$$
E_{\theta}=i_{n} \frac{k e^{-i k r}}{4 \pi r} \operatorname{seno} \theta\left[\int_{-1 / 2}^{+1 / 2} I(z) e^{i k z \operatorname{cosen} o \theta} d z\right]
$$

\section{- Nanoantenas}

Los nanotubos poseen algunas propiedades eléctricas particulares en comparación con las de un alambre de cobre de la misma longitud y diámetro. Un punto de partida es la conductividad de los nanotubos, cuya medición es aproximadamente dos veces la del cobre: $10^{8} \mathrm{Sm}^{-1}$ para un nanotubo de carbono con un diámetro de $1,5 \mathrm{~nm}$ y $5,8 \times 10^{7} \mathrm{Sm}^{-1}$ para una conductividad superior a la del cobre. Se encuentra además que la velocidad de propagación de la onda en los nanotubos de carbono comienza alrededor de los 1/50th de la velocidad de la luz y en las uniones se reduce a 1/100th cuando la frecuencia de excitación se aproxima a la resonancia (Boudenot, 2008) y (Burke, et al., 2005).

Por la longitud que poseen los nanotubos de carbono es posible considerar, de modo realista, antenas para comunicación inalámbrica en GHz y THz, tanto en el "macro-mundo" por una parte, como en el de los "nanodispositivos" por la otra (Adnan, et al., 2016), (Mohammad, 2017), (Zakrajsek, et al., 2017), (Ziaei, et al., 2008) y (Eltresy, et al., 2017).

Para los autores en (Boudenot, 2008) ha sido un reto analizar la viabilidad de las antenas en la frecuencias de los GHz, usando nanotubos de carbono como dipolos resonantes (Boudenot, 2008). Es importante destacar que probablemente debido a las propiedades eléctricas que poseen los nanotubos, pueda limitarse considerablemente la eficacia potencial de la antena. Dichos problemas se clasifican como las pérdidas óhmicas y la elevada frecuencia de "relajación" que posee el electrón (Boudenot, 2008). 
Así mismo, el diseño de las nanoantenas se ha inspirado bajo las características y propiedades de algunos metamateriales, donde los resultados obtenidos han sido igual de sorprendentes a los derivados del carbón. Un ejemplo de ello se refleja en (Links, 2011), donde investigadores de la Universidad de Brest y la Corporación de Investigación Rennes Thomson de I+D en Francia, desarrollan una antena miniatura para operación en la banda de UHF. Es un diseño que utiliza una clase de metamaterial y cuyas propiedades magneto-dieléctricas sirven para el soporte de la antena, además de una red de adaptación pasiva para la sintonización de frecuencia y una adaptación de la impedancia sobre toda la banda de UHF. La operación de esta antena fue simulada y medida en una terminal nómada y sus resultados fueron adecuados para aplicaciones en video (Links, 2011).

\section{- Nanotubos de carbono}

Entre las nanoestructuras más utilizadas hoy en día, los nanotubos de carbono (CNT) en aplicaciones de radio, han ido aumentando, al igual que la sincronización con las nanoredes NNW (Nanonetworks), en los que procedimientos básicos como la incorporación de una antena, un sintonizador, un demodulador, y un amplificador de radio, se logran con un solo nanotubo de carbono. Aquí se refleja la recepción de la misma señal, el "tuning", la amplificación y los procesos electromecánicos de demodulación (Dressler, 2016) y (Jensen, et al., 2007). De esta forma la frecuencia de la resonancia de los nanotubos de carbono debería coincidir con la portadora WC (que es afectada por la longitud de los nanotubos). Otro de los enfoques se basa en la modulación FM (transmisor), mediante la aplicación de señales con información hacía el electrodo externo (Vtensión) (Dressler, 2016) y (Jensen, et al., 2007).

\section{- Nanotubos en aplicaciones de radio}

Han pasado ya alrededor de dos décadas cuando en 1991 se descubrieron los nanotubos de carbono, y es ahora donde cobran mayor impacto las propiedades eléctricas que poseen, donde su futuro promete avances significativos en el ámbito electrónico (Jensen, et al., 2007). De igual forma, sus propiedades térmicas y mecánicas se convierten en atractivos para una amplia gama de campos de aplicación, como líneas de transmisión en la nanoescala, nanocomponentes activos y pasivos tales como transistores o interruptores en circuitos de alta densidad y en las biotecnologías (Boudenot, 2008).

Una de las soluciones para operar las nanotecnologías en el campo actual se logra estableciendo la comunicación y el intercambio de datos a altas velocidades entre los diferentes dispositivos $\mathrm{u}$ elementos mediante las nanoantenas para comunicaciones inalámbricas, las cuales están soportadas por nanotubos de carbono. Una muestra de ello son las redes de telecomunicaciones con los conmutadores de radio frecuencia, como pieza clave para llevar a cabo las siguientes subfunciones:

-Conmutación de transmisión y recepción. -Conmutación en la banda (celulares).
-Desfasadores en antenas para orientar el haz electrónico (e.g. antenas de satélite, radares aéreos) (Boudenot, 2008).

Para los diseñadores de circuitos en el campo de las microondas, en el ingreso de los NEMS (Nano Electro Mechanical Systems) se prevén desarrollos adicionales, debido a que permiten llevar a cabo conmutaciones de muy alto rendimiento mediante comandos electrostáticos. Rendimiento que se manifiesta con una baja pérdida de inserción y un alto aislamiento, a su vez, un tiempo de conmutación inferior a $0,1 \mathrm{~ms}$, cuyo orden de magnitud es más bajo que el estado de la técnica para interruptores NEMS. Tensión de servicio por debajo de $1 \mathrm{~V}$, capacidad de manejo en las señales de alta frecuencia de radiofrecuencia, bajo consumo de corriente, bajo costo y alta densidad de integración (Boudenot, 2008) y (Ziaei, et al., 2008).

Los NEMS se caracterizan por sus pequeñas dimensiones, que han llegado a ser relevantes para la función de otros dispositivos. Su tamaño puede llegar a ser de unos cientos a unos pocos nanómetros. Las nuevas propiedades físicas resultado de estas pequeñas dimensiones, pueden llegar a dominar el funcionamiento de 
los demás dispositivos y los nuevos enfoques de fabricación pueden llegar a realizarse con base en ellos. Es por esto que la implementación de nanotubos de carbono en los NEMS se ha convertido en algo interesante, debido a sus propiedades eléctricas y mecánicas (Stebunov, 2012), (Boudenot, 2008) y (Ziaei, et al., 2008).

Los estudios obtenidos en (Habibpour, et al., 2016) analizan la demodulación de una señal de encendido y apagado multi-Gb/s a través de un portador a $96 \mathrm{GHz}$ utilizando un transistor FET de grafeno a una escala de 250 nm como detector de potencia con polarización a cero, demostrando así el potencial que tienen este tipo de dispositivos y los niveles de comunicación que pueden obtenerse a dichas frecuencias. De igual forma ocurre en (Mao, et al., 2016) y (W., et al., 2017) con la implementación de grafeno para lograr osciladores GTFET (túnel de grafeno FET) en donde se analizan parámetros clave para el dispositivo tales como la frecuencia, la potencia de salida, funcionamiento, oscilación máxima, potencia máxima, relación entre frecuencia y potencia y rendimiento, y todo encaminado a lograr una comunicación eficaz.

\section{- Nodos de sensores de nanotubos de carbono}

Investigadores en (Akan, 2017) documentan que para lograr ésta aplicación, en un principio es importante la observación de muchos nanodispositivos en sus primeras etapas de desarrollo. Esto con el fin de aprovechar las características únicas de cada nanocomponente, impuestas en la arquitectura de su sistema unificado.

En la Figura 6 se visualiza un modelo de un nodo de sensor a base de nanotubos de carbono compuesto por cada uno de sus componentes en una escala nanométrica. Cada uno ejecutando una función específica y primordial para un correcto funcionamiento. Algunos de sus componentes más destacados son:

- Un nanoreceptor

- Una nanounidad de poder.

- Un nanoprocesador y una nanomemoria.

- Y unidades alternas de nanosensores.

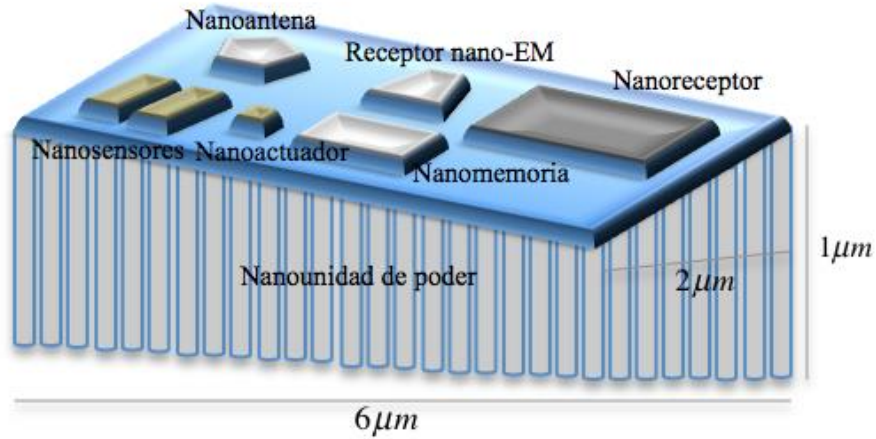

Figura. 6. Modelo de un nodo de sensor con nanotubos de carbono compuesto por cada uno de sus nanocomponentes.

\section{- Redes Ad Hoc (CANET) basadas en CNT}

Al analizar este tipo de redes se presentan algunos interrogantes tales como:

- El dominio sobre las redes Ad Hoc en las comunicaciones inalámbricas es un desafío bastante extremo.

- La baja potencia en la señal del nanotransmisor, es equivalente a un muy limitado alcance en radio.

- Son extremadamente propensas al ruido térmico y a la decoloración.

- Requieren de alta potencia para la radio con nanotubos, lo equivalente a otro desafío más para realizarlo (Atakan, et al., 2010) y (Boudenot, 2008).

Es por ello que en la Figura 7 se visualiza un sistema de nanonodos cuya transmisión y recepción se realiza en forma inalámbrica con un objetivo principal y es la detección de moléculas antígenas a través de biosensores. Paralelamente se encuentran otro tipo de nanosensores como los embebidos con una aplicación puntual en los sistemas de monitoreo medico. Construídos con materiales piezoeléctricos que operan mediante vibraciones ultrasónicas generadas por pequeñas fuentes generadoras de energía eléctrica. Los estudios en (Donohoe, et al., 2016) demuestran un rendimiento óptimo y operan adecuadamente si se utilizan como nanosensores al interior del cuerpo humano. 


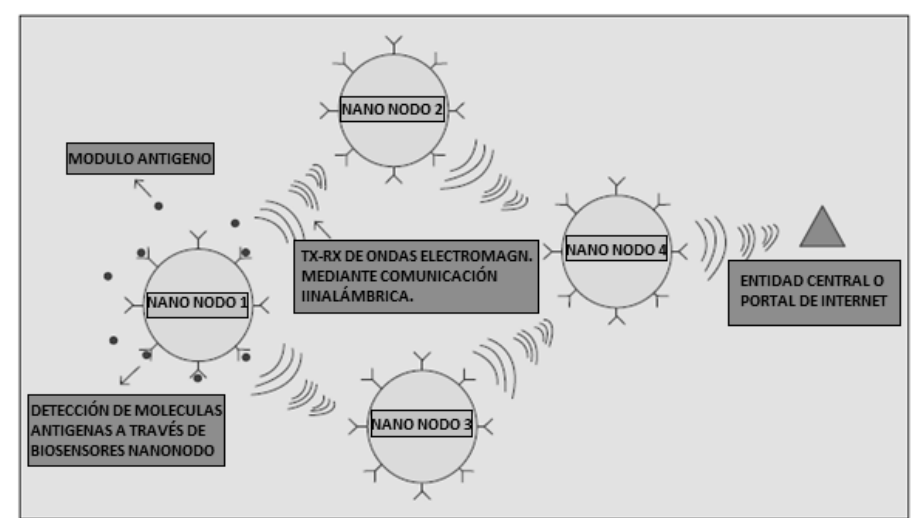

Figura. 7. Aplicación CANET: Red de biosensores en escala nanométrica.

\section{IV. ÚLTIMOS AVANCES EN NANOCOMUNICACIONES}

\section{A. Osciladores mecánicos de grafeno con frecuencias sintonizables}

A través de las investigaciones dirigidas por (Chen, et al., 2013) y (Nguyen, 2005) de la Universidad de Columbia, fue posible el diseño, construcción y puesta en marcha de un nanooscilador mecánico de grafeno, para la sintonización de frecuencias en FM.

Los osciladores que producen señales periódicas continuas de potencia en corriente directa son el centro de los sistemas de comunicaciones modernos, con aplicaciones versátiles que incluyen referencias de temporización y moduladores de frecuencia (Chen, et al., 2013) y (Hajimiri, et al., 1999)

Así mismo, la estructura de los osciladores convencionales suele estar conformada por resonadores mecánicos macroscópicos, algunos con cristales de cuarzo (los cuales requieren un mayor espacio y por lo general fuera del chip). A diferencia de estos osciladores, construidos en micrómetros de tamaño, los resonadores nanomécanicos de grafeno de un grosor atómico alcanzan frecuencias que pueden ser electrostáticamente afinadas hasta en un $14 \%$, en las que un movimiento mecánicamente autosostenible se genera $y$ transduce a temperatura ambiente en estos osciladores usando un simple circuito eléctrico (Hajimiri, et al., 1999) y (Nguyen, 2005).

Los osciladores controlados por voltaje (prototipo de grafeno) exhiben cierta estabilidad de frecuencia y ancho de banda para una modulación eficiente, con el fin de modulares señales portadoras de radiofrecuencia. En demostración, los investigadores de la Universidad de Columbia implementaron un oscilador de grafeno como elemento activo, modulando una señal y logrando una transmisión eficiente en términos de audio. Ésta fue transmitida en modulación FM a una frecuencia de 100 MHz. Su recepción se logró a través de un radio convencional con un poco de estática (Chen, et al., 2013).

\section{B. Comunicaciones cuánticas a nanoescala}

Las nanocomunicaciones se han ido definiendo como aquella área de la investigación que se encarga de la búsqueda de medios eficaces de comunicación para los futuros nanodispositivos, los cuales están previstos para operar en una amplia gama de áreas de aplicación. En la actualidad, la investigación en nanocomunicaciones se divide en dos corrientes principales: Nanocomunicaciones EM y Nanocomunicaciones moleculares (Burhan, 2017), (Niamien, et al., 2011) y (Malak, et al., 2016).

\section{- Comunicaciones electromagnéticas (EM) en THz}

Las nanocomunicaciones electromagnéticas utilizan ondas electromagnéticas como portadoras de información, muy similares a una comunicación clásica. Sin embargo, por la extrema escasez de recursos y por los efectos cuánticos de los mismos materiales, los métodos clásicos no son posible aplicarlos directamente en el dominio nano. Lo que nos direcciona a aplicar materiales y técnicas novedosas (Burhan, 2017), (Hanson, 2005), (Links, 2011), (Pankaj, et al., 2017), (Zainuddin, et al., 2017) y (Malak, et al., 2016).

Es así como el grafeno se ha establecido como un material que promete un sin número de aplicaciones, lo que lo ha convertido en foco de investigación e implementación en los últimos años. A nivel de las nanocomunicaciones se han registrado diversos aportes a su desarrollo. Es el caso de los CNT cargados, los cuales resuenan a través de la excitación de una "ola" electromagnética. Y su distancia a través de los cambios de estado con base en la amplitud de la onda. La variación de la distancia también modifica las propiedades de la emisión de los campos del CNT que resultan de la misma variación en la corriente. Concluyendo así que sería posible utilizar un nanotubo de carbono como 
antena y como demodulador al mismo tiempo (Links, 2011) y (Malak, et al., 2016).

\section{- Comunicación molecular}

La comunicación molecular se define como aquella técnica de comunicación natural utilizada por organismos vivos (vía feromonas) y está concebido para convertirse, en un futuro, en un método viable para implementación en nanodispositivos. Lo que se traduce en que la concentración de una molécula en las proximidades del receptor se puede utilizar para comprender el transmisor molecular en los bits enviados (Hanson, 2005) y (Links, 2011). Una muestra de ello se relaciona en (Felicetti, et al,.2015) donde un análisis de las comunicaciones moleculares basadas en difusión, se caracterizan por medio del movimiento browniano de las mismas moléculas de la señal durante la trayectoria desde una nanomáquina transmisora hacía una receptora. Centrándose así en mejorar la calidad y la capacidad de recibir señales biológicas.

\section{- Comunicaciones cuánticas}

Las comunicaciones cuánticas se basan en la transferencia de pares entrelazados de un lugar a otro, con la ayuda de intercambio, repetición y purificación. Por otro lado, la interferencia cuántica o paralelismo cuántico proporciona una inmensa potencia de cálculo, sobre todo en la codificación de origen, donde en lugar de entradas individuales, se estructura información sobre todo el contenido requerido, es decir, se consolida y transporta todo en un solo canal (Hanson, 2005), (Links, 2011) y (Volz, et al., 2007).

\section{CONCLUSIONES Y COMENTARIOS}

En este artículo nosotros expusimos una breve introducción sobre lo que es la nanotecnología y el despliegue de las nanocomunicaciones entorno a ella. Una mirada enfocada a conocer aquellos nanomateriales y sus propiedades, que hoy día forman un pilar fundamental en la investigación y desarrollo de nuevos nanodispositivos en el ámbito de las comunicaciones.

La intervención de la nanotecnología en las comunicaciones es fundamental para lograr desarrollar soluciones que tienen como objetivo ayudar a suplir las necesidades de nuestra sociedad y a fortalecer la capacidad tecnológica.

Los avances con grafeno y nanotubos de carbono que se tienen hasta ahora y los desarrollos en comunicaciones de tipo cuántico y molecular son una pequeña muestra de hasta dónde se puede llegar con esta fusión de tecnologías. Nanomateriales con altas tasas de conductividad eléctrica, térmica y moldeabilidad mecánica permiten construir desde el más sencillo circuito, hasta el más pequeño transmisor para modulaciones en FM transmitiendo en frecuencias de hasta $100 \mathrm{MHz}$, mediante un oscilador incrustado en láminas de grafeno a una escala nanométrica.

\section{REFERENCIAS BIBLIOGRÁFICAS}

Adnan, S. \& Goni, O., 2016. Graphene nanoribbon based antenna for terahertz band communication. In 2nd International Conference on Electrical Information and Communication Technologies, EICT 2015. Khulna, Bangladesh: IEEE, pp. 389-393. Available at: http://ieeexplore.ieee.org/document/7391982/.

Atakan, B. \& Akan, O., 2010. Carbon nanotube-based nanoscale ad hoc networks. IEEE Communications Magazine, 48(6), pp.129-135. Available at: http://dl.acm.org/citation.cfm?id=1824644.

Atsuko Sekiguchi, et al., 2017. Stretchable and robust transistor of single wall carbon nanotube, gel and elastomeric materials. In Electronics Packaging (ICEP), 2017 International Conference on. Yamagata, Japan: IEEE, pp. 19-22. Available at: http://ieeexplore.ieee.org/document/7939419/.

Bartolomé, F. et al., 2007. Nuevos materiales en la sociedad del siglo XXI CSIC, ed., Available at: http://www.csic.es/buscar?p_p_state=maximized\&p_p_lif ecycle $=1 \&$ _contentviewerservice_WAR_alfresco_packpo rtlet_struts_action=\%2Fcontentviewer $\% 2$ Fview\&p_p_id= contentviewerservice_WAR_alfresco_packportlet\&_conte ntviewerservice_WAR_alfresco_packportlet_nodeRef $=$.

Bernholc, J. et al., 2002. Mechanical and Electrical Properties of Nanotubes. Annual Review of Materials Research, 32, pp.347-375. Available at: http://www.annualreviews.org/doi/pdf/10.1146/annurev.m atsci.32.112601.134925. 
Stebunov, Y., 2012. Graphene nanoribbon based AM demodulator of terahertz radiation. pp.5-8. Nano/Micro Engineered and Molecular Systems (NEMS), 2012 7th IEEE International Conference on. Available at: http://ieeexplore.ieee.org/document/6196881/.

Boudenot, J.-C., 2008. New concepts for nanophotonics and nano-electronics: From transistor to nanotube. C.R. Physique, 9(1), pp.41-52. Available at: http://www.sciencedirect.com/science/journal/16310705/9 $/ 1 ? \mathrm{sdc}=1$.

Burhan Gulbahar, G.M., 2017. Nanoscale optical communications modulator and acousto-optic transduction with vibrating graphene and resonance energy transfer. In Communications (ICC), 2017 IEEE International Conference on. Paris, France: IEEE, pp. 21-25. Available at: http://ieeexplore.ieee.org/document/7997036/.

Burke, P.J., Yu, Z. \& Rutherglen, C., 2005. Carbon nanotubes for RF and microwaves. European Gallium Arsenide and Other Semiconductor Application Symposium, GAAS 2005, pp.4-7. Available at: chttp://amsacta.unibo.it/1470/1/GA052485.PDF.

Burke, P.J., Yu, Z. \& S, L., 2004. Quantitative Theory of Nanowire and Nanotube Antenna Performance. Mesoscale and Nanoscale Physics, p.15. Available at: https://arxiv.org/pdf/cond-mat/0408418.pdf.

Chen, C. et al., 2013. Graphene mechanical oscillators with tunable frequency. Nat Nanotechnol, 8(12), pp.923927. Available at: http://dx.doi.org/10.1038/nnano.2013.232.

Diego Scaccabarozzi, et al., 2017. A preliminary study on self-sensing composite structures with carbon nanotubes. In Metrology for AeroSpace (MetroAeroSpace), 2017 IEEE International Workshop on. Padua, Italy: IEEE, pp. 21-23. Available at: http://ieeexplore.ieee.org/document/7999613/.

Donohoe, M. et al., 2016. Powering In-Body Nanosensors With Ultrasounds. IEEE Transactions on Nanotechnology, 15(2), pp.151-154. Available at: http://ieeexplore.ieee.org/document/7358144/.
E. Abad, 2005. NanoDictionary, Available at: http://www.colbas.org/pub.htm.

Falko Dressler, 2016. Biologically-inspired and Nanoscale Communication and Networking. In ACM Distinguished Speaker Program and IEEE International Conference on Advances in ICT for Emerging Regions. Negombo, Sri Lanka. Available at: http://www.ccslabs.org/ dressler/curriculum-vitae.shtml.

Felicetti, L., Femminella, M. \& Reali, G., 2015. Smart Antennas for Diffusion-based Molecular Communications. Proceedings of the Second Annual International Conference on Nanoscale Computing and Communication, (September), p.27:1--27:6. Available at: http://dl.acm.org/citation.cfm?id=2800817.

Foresight Institute, 2014. Advancing beneficial nanotechnology. Available at: http://www.foresight.org/nano/index.html [Accessed March 14, 2014].

García, J., 2010. Grafeno, el material del futuro. Available at: https://www.grafeno.com/que-es-el-grafeno.

Gou, Y. et al., 2011. Broadband microstrip antennas using complementary metamaterials structure. In Proceedings of 2011 Cross Strait Quad-Regional Radio Science and Wireless Technology Conference, CSQRWC 2011. IEEE, pp. 530-533. Available at: https://www.infona.pl/resource/bwmeta1.element.ieee-art000006037003 ?locale $=$ en.

Habibpour, O. et al., 2016. Graphene FET gigabit ONOFF keying demodulator at $96 \mathrm{GHz}$. IEEE Electron Device Letters, 37(3), pp.333-336. Available at: http://ieeexplore.ieee.org/document/7378844/.

Hajimiri \& Thomas H. Lee, 1999. THE DESIGN OF LOW NOISE OSCILLATORS 1999th ed., Springer. Available http://www.ncbi.nlm.nih.gov/pubmed/15003161\%5Cnhttp ://cid.oxfordjournals.org/lookup/doi/10.1093/cid/cir991\%5 Cnhttp://www.scielo.cl/pdf/udecada/v15n26/art06.pdf\%5 Cnhttp://www.scopus.com/inward/record.url?eid=2-s2.084861150233\&partnerID=tZOtx3y1. 
Hanson, G.W., 2005. Fundamental transmitting properties of carbon nanotube antennas. IEEE Transactions on Antennas and Propagation, 53(11), pp.3426-3435. Available at: https://bwn.ece.gatech.edu/nanos/papers/nano_antennas classical_analysis.pdf.

Jensen, K. et al., 2007. Nanotube radio. Nano Letters, 7(11), pp.3508-3511. Available at: http://pubs.acs.org/doi/abs/10.1021/n10721113.

Links, D.A., 2011. Nanoscale COMMUNICATION Artech House, ed. , pp.3609-3612. Available at: https://books.google.com.co/books?id=YsuhQJ3mSh8C\& $\mathrm{dq}=$ Nanoscale + Heat + Communication $\& \mathrm{hl}=\mathrm{es} \&$ source $=\mathrm{gbs}$ _navlinks_s.

Mao, X. et al., 2016. Characterization of Graphene TFET for Subterahertz Oscillator. IEEE Transactions on Electron Devices, 63(7), pp.2956-2962. Available at: http://ieeexplore.ieee.org/document/7479521/.

Mohammad Ali Shamble, R.S., 2017. Waveguide-fed graphene-based hybrid plasmonic patch antenna. In Electrical Engineering (ICEE), 2017 Iranian Conference on. Tehran, Iran: IEEE, pp. 2-4. Available at: http://ieeexplore.ieee.org/document/7985302/.

Moon, J.S. et al., 2011. Graphene transistors for RF applications: Opportunities and challenges. 2011 International Semiconductor Device Research Symposium (ISDRS), 30, pp.1-2. Available at: http://ieeexplore.ieee.org/document/6135155/.

N.A. Eltresy, H.A. Malhat, S.H.Z.-D., 2017. A rhombic nanoantenna for infrared detection applications. In Radio Science Conference (NRSC), 2017 34th National. Alexandria, Egypt: IEEE, pp. 13-16.

Nguyen, C.T.-C., 2005. MEMS Technologies for Timing and Frequency Control. Ifcs, 54(2), pp.1-11. Available at: http://escholarship.org/uc/item/19m6207z.

Niamien, C. et al., 2011. Ultra-miniature UHF antenna using magneto-dielectric material. Electronics Letters, 47(5), pp.300-301.
Ozgur Baris Akan, 2017. Researcher, Istanbul, Turkey. Available at: http://home.ku.edu.tr/ akan/.

Pankaj Singh, et al., 2017. Preamble-based synchronisation scheme for electromagnetic wireless nanocommunications. IET Communications, 11(7), pp.511. Available at: http://ieeexplore.ieee.org/document/7933168/.

Ramsden, J., 2009. Essentials of Nanotechnology Download free books at, Ventus Publishing. Available at: http://bookboon.com/en/nano-technologyebook\#download.

Speech, K., 2012. Metamaterials - From New Concepts to Applications. IEEE "Metamaterials-From new concepts to applications." Electromagnetics; Applications and Student Innovation (iWEM), pp.2009-2010. Available at: http://ieeexplore.ieee.org/document/6320340/authors.

The National Nanotechnology, 2001. National Nanotechnology Initiative. JNCI Journal of the National Cancer Institute, 93(24), pp.1-2. Available at: http://www.nano.gov/.

Volz, S. \& Carminati, R., 2007. Microscale and nanoscale heat transfer CRC Press, ed., Available at: https://books.google.com.co/books?id=InKmCwAAQBAJ $\& \mathrm{dq}=$ Nanoscale + Heat + Communication $\& \mathrm{hl}=\mathrm{es} \&$ source $=\mathrm{g}$ bs_navlinks_s.

W. Wei, et al., 2017. Graphene field effect transistors with optimized contact resistance for current gain. In Device Research Conference (DRC), 2017 75th Annual. South Bend, IN, US: IEEE, pp. 25-28. Available at: http://ieeexplore.ieee.org/document/7999420/.

Zainuddin, M.A., Dedu, E. \& Bourgeois, J., 2017. Simple and energy efficient image compression for pulsebased communication in thz band. In Proceedings International Conference on Advanced Information Networking and Applications, AINA. Taipei, Taiwan: IEEE, pp. 112-119. Available at: http://ieeexplore.ieee.org/document/7920898/.

Zakrajsek, L. et al., 2017. Design of Graphene-based Plasmonic Nano-antenna Arrays in the Presence of Mutual 
https://doi.org/10.25054/issn.2216-1325

Coupling. Antennas and Propagation (EUCAP), 2017 11th European Conference on, pp.1390-1394. Available at: http://ieeexplore.ieee.org/document/7928818/.

Ziaei, A. et al., 2008. Review of two microwave applications of carbon nanotubes: nano-antennas and nanoswitches. Proceedings of SPIE, 6931(1), p.69310R69310R-12. Available at: http://www.sciencedirect.com/science/article/pii/S163107 0508000108\#!

Cui, T.J, 2012. Metamaterials - From new concepts to applications. Electromagnetics; Applications and Student Innovation (iWEM), 2012 IEEE International Workshop on. 6-9 Aug. 2012, IEEE. Available at: http://ieeexplore.ieee.org/document/6320340/

Malak, D and Ramezani, $\quad \mathrm{H}$ and Kocaoglu, M and Akan, OB., 2016. Diversity in diffusion-based molecular communication channel with drift. Communications (ICC), 2016 IEEE International Conference on, p.1-6. IEEE. Available at: https://scholar.google.com/citations?view_op=view_citati on\&hl=es\&user=sKT2oLkAAAAJ\&citation_for_view=s KT2oLkAAAAJ:0KyAp5RtaNEC 